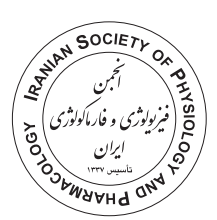

\title{
Combination effects of gallic acid and cyclosporine A during ischemia/ reperfusion on rat electrocardiogram parameters and arrhythmia
}

\author{
Najmeh Sadeghi' ${ }^{1}$, Samira Saadatfard ${ }^{2}$, Mahin Dianat ${ }^{3}$, Hassanali Abedi ${ }^{4}$, Khatereh Dehghani $^{5^{*}}$ (ID
}

1. Sirjan School of Medical Sciences, Sirjan, Iran

2. Research Center for non. Communicable Diseases, Jahrom University of Medical Sciences, Jahrom, Iran

3. Department of Physiology, Faculty of Medicine, Ahvaz University of Medical Sciences, Ahvaz Iran

4. Department of Physiology, Faculty of Medicine, Jahrom University of Medical Sciences, Jahrom, Iran

5. Department of Cardiology, Faculty of Medicine, Jahrom University of Medical Sciences, Jahrom, Iran

\begin{abstract}
Introduction: Myocardial ischemia leads to electrical disturbance in the heart because of reactive oxygen specious. This study aimed to investigate the effects of gallic acid and cyclosporine $\mathrm{A}(\mathrm{Cs} \mathrm{A})$ together on electrocardiogram parameters in myocardium following ischemia - reperfusion $(\mathrm{I} / \mathrm{R})$ in isolated hearts.

Methods: In this research, 50 Wistar rats weighing 250-300g were randomly divided into the 5 following groups: control, sham and gallic acid $(7.5,15$ and $30 \mathrm{mg} / \mathrm{kg})$ in combination with $\operatorname{CsA}(0.2 \mu \mathrm{M})$. On the eleventh day, the hearts were removed and perfused with Krebs solution and ischemia was induced for $30 \mathrm{~min}$. Then, cyclosporine was administered for $10 \mathrm{~min}$ at the 10 minutes before reperfusion and 10 minutes the beginning of reperfusion. By placing the electrode, the parameters of RR, PR, QT, TpeakTend, JT and QTcB interval, ST elevation, R, P, Q, S, T amplitude were recorded before ischemia and during reperfusion. Results: This study showed that RR, JT, interval, p duration, ST elevation and PVC numbers of control were increased during ischemia compared with sham and decreased using gallic acid $(7.5,15$ and $30 \mathrm{mg} / \mathrm{kg})$ in combination with CsA. In addition, P, R, S, T amplitude during the ischemia were decreased in control compared with sham and increased with gallic acid $(15 \mathrm{mg} / \mathrm{kg})$ in combination with CsA.

Conclusion: In conclusion, the optimal combination of both drugs decreased arrhythmia occurrence while increased electrical velocity of conduction and wave amplitudes in isolated myocardium after ischemia reperfusion injury.
\end{abstract}

\author{
Keywords: \\ Gallic acid \\ Cyclosporine A \\ Ischemia reperfusion injury
}

\section{Introduction}

At present, cardiovascular diseases account for nearly half of no communicable diseases in the world. During the last years, ischemic heart diseases as the global leading cause of death, has been accounted for 17.3 million deaths per year in the world and 2726 per day in Iran (Adib et al., 2020; Moradi-Lakeh et al., 2017). Arrhythmias or dysrhythmias are applied

\footnotetext{
* Corresponding author: Khatereh Dehghani, dkh.dehghani@gmail.com

Received 27 January 2020; Revised from 26 October 2020; Accepted 23 November 2020
}

Citation: Sadeghi N, Saadatfard S, Dianat M, Abedi H, Dehghani K. Combination effects of gallic acid and cyclosporine A during ischemia/ reperfusion on rat electrocardiogram parameters and arrhythmia. Physiology and Pharmacology 2021; 25: 162-170. http://dx.doi.org/10.32598/ppj.25.2.80 
to any kind of rhythm except normal sinus rhythm. Arrhythmia can be induced by many factors including abnormal automaticity, myocardial ischemia, electrolyte abnormalities, metabolic disorders, valvular heart disease, congenital heart disease and even some medications (Zheng et al., 2020). Myocardial ischemia occurs when the supply/demand ratio of blood in myocardial is insufficient. Ischemia induced hypoxia leads to the tissue necrosis as well as changes in the pattern of metabolism and electrical rhythm (Buja and Weerasinghe, 2010; Ebner, 2020).

In the clinic, coronary artery bypass surgery, angioplasty and thrombosis removal are performed to restore the coronary blood flow and myocardial salvage before the progress of irreversible complications. Ischemia reperfusion injury (I/R) causes contractile function impairment of the heart, biochemical and structural changes, arrhythmias, contracture myofibrillar, irreversible myocardial necrosis due to the generation of reactive oxygen species (ROS), impaired calcium homeostasis as well as $\mathrm{pH}$ changes during the ischemic period (Buja and Weerasinghe, 2010; White, 2020; Yellon and Hausenloy, 2007). Besides, the increased production of mitochondrial ROS in ischemia opens the mitochondrial pores (mitochondrial permeability transition pore, mPTP) that intensify the production of ROS and create a vicious cycle (Giugliano, 2000; Pagliaro et al., 2019; Yellon and Hausenloy, 2007).

Itwas reported in a research that gallic acid (a metabolite propyl gallate), which possesses potent antioxidant properties by protects lysosomes membrane and heart muscle morphology against oxidative stress and reduces the myocardial necrosis (Priscilla and Prince, 2009; Prince et al., 2009; Hung et al., 2001; Priscilla and Prince, 2009). In addition to its antioxidant properties, gallic acid is anti-arrhythmic.

According to Dianat et al. (2013) report, short and long term consumption of gallic acid can prevent from ventricular fibrillation (VF), V-tach and premature ventricular pulses against $\mathrm{CaCl} 2$-induced arrhythmia in rat.

Also, mPTP inhibitors such as cyclosporine A (CsA) by improving calcium homeostasis as well as reducing the infarct size up to $60 \%$ (Hausenloy et al., 2003; MarinGarcia and Goldenthal, 2004) can limit the myocyte necrosis and apoptosis. According to a past study, acute myocardial ischemia reduces dephosphorylation of connexin 43 which delays electrical activity of myocardial and increases the anisotropic conductivity. As a result, myocardium will be susceptible to ventricular arrhythmias. During ischemia, the expression of potassium channel $\left(\mathrm{KV}_{4}\right)$ and its flow $\left(\mathrm{I}_{\mathrm{to}}\right)$ are reduced which delays the repolarization phase and transient potassium current. Therefore, VF susceptibility increases and heart rate variability decreases (Kario, 2010).

In recent years, antioxidants and $\mathrm{MPTP}$ inhibitors have been used to reduce the effects of $I / R$ injuries and each revive the myocardia against MI to only $40-60 \%$ (Hausenloy et al., 2003; Priscilla and Prince, 2009). Improved electrocardiographic (ECG) recordings has become an attractive approach for the diagnosis of myocardial infarction. ST-segment elevation, QTprolongation, $\mathrm{R}$ wave amplitude are well-recognized changes in ECG recording in patients with myocardial infarction. The early prediction of myocardial tissue subjected to the risks of myocardial infarction is of significant importance for prevention or precaution against adverse outcome caused BY ischemic condition (Sun et al., 2013). So far, no study has been carried out to investigate the effect of two combined together drugs on ECG parameters' changes in myocardium after $I / R$ in isolated hearts. So, using the combination of CsA and gallic acid as an antioxidant and MPTP inhibitor, we decided to study the protective effects of two combined together drugs on ECG parameters' changes and arrythmia in ischemia condition.

\section{Materials and methods}

Total of 50 male Wistar rats weighing 250-300g were used in this experimental study. Animals were kept in appropriate condition of temperature $\left(21 \pm 2^{\circ} \mathrm{C}\right)$, lightdark cycle (12/12 hour) and free access to regular diet and water. Finally, they were randomly divided into 5 groups as follows: control and sham group that received saline, 3 groups that received a combination of gallic acid $(7.5,15$ and $30 \mathrm{mg} / \mathrm{kg}$ and cyclosporine $\mathrm{A}(0.2 \mu \mathrm{M})$. Gallic acid and cyclosporine were purchased from sigma.

The control group had not undergone protocol while the other three groups (8-10 in each) underwent $I / R$ protocol as follows: Sham: did not receive any treatment; CsA: received cyclosporine $(0.2 \mu \mathrm{M})$ (Xie and $\mathrm{Yu}$, 2007); G7.5: received $7.5 \mathrm{mg} / \mathrm{kg}$ gallic acid (Prince et al., 2009); G7.5+CsA: received $7.5 \mathrm{mg} / \mathrm{kg}$ gallic acid + CsA; G15: received $15 \mathrm{mg} / \mathrm{kg}$ gallic acid; G15+CsA: 
received $15 \mathrm{mg} / \mathrm{kg}$ gallic acid $+\mathrm{CsA}$; G30: received $30 \mathrm{mg} / \mathrm{kg}$ gallic acid; G30+CsA: received $30 \mathrm{mg} / \mathrm{kg}$ gallic acid + CsA.

Gallic acid was directly dissolved in normal saline and was administered with gavage needle once a day for 10 days at 8 o'clock every morning (Prince et al., 2009). All groups experienced I/R protocol except the control group. Duration of protocol was 10 days and gavage volume was $1 \mathrm{ml}$ in all groups. It should be noted that isolated hearts were perfused with cyclosporine $(0.2 \mu \mathrm{M})$ for 10 minutes before the induction of ischemia and 10 minutes at the start of reperfusion (Badavi et al., 2014). The control group did not receive protocol. Electrocardiographic parameters such as RR, PR, QT, TpeakTend, JT, QTc interval, ST elevation, R, P, Q, S, T amplitude and heart rate were computed at pre-ischemia, ischemia and reperfusion period.

\section{Preparation and perfusion of isolated hearts}

Animals were anesthetized using intraperitoneal injection of ketamine $(50 \mathrm{mg} / \mathrm{kg})$ and xylazine $(2 / 5 \mathrm{mg} /$ $\mathrm{kg})$ at 10 o'clock in the morning. To prevent blood clotting, heparin was injected (1000 units, Caspian, Iran) intraperitoneally at $20 \mathrm{~min}$ before induction of anesthesia. The tracheas were cannulated and the animals were ventilated with room air using a rodent ventilator (Harvard mouse/rat Ventilator Model 687. USA, 1901). In the following, chests were opened and heart aortic cannulas were inserted. Immediately, hearts were perfused with Krebs-Hanslate bicarbonate solution at constant pressure $(60-70 \mathrm{mmHg})$ and at $37^{\circ} \mathrm{C}$. Krebs solution composition was as follows: $\mathrm{NaCl}$ : 118.4mM, KCl: $4.7 \mathrm{mM}, \mathrm{MgSO}_{4}: 1.21 \mathrm{mM}, \mathrm{KHPO}_{4}$ : $1.2 \mathrm{mM}, \mathrm{NaHCO}_{3}: 25 \mathrm{mM}, \mathrm{CaCL}_{2}: 2.5 \mathrm{mM}$ and glucose: $11.1 \mathrm{mM}$. This buffer was gassed with a combination of $95 \%$ oxygen and $5 \% \mathrm{CO}_{2}$ mixture to reach $\mathrm{pH}=7.4$. The temperature of this solution was preserved at $37 \pm 0.5^{\circ} \mathrm{C}$. Heart rate and ECG parameters (Lead II) were recorded retrospectively in three times (before, during and after the ischemia induction) using langendorff apparatus (AD Instruments, and Australia, ML176-V) .

After fixing the hearts in the cardiac chamber, the experiment was done on them for $105 \mathrm{~min}$. Initially, diastolic pressure was maintained at $1-6 \mathrm{mmHg}$. Then, $15 \mathrm{~min}$ for equilibrium during which the heart rate and other parameters were recorded, after that $30 \mathrm{~min}$ for induction of ischemia and $60 \mathrm{~min}$ for reperfusion
(Klawitter et al., 2002).

ECG was recorded by attaching three spring clip electrodes directly to the heart surface (MLA1210, AD Instruments, and Australia). The signals from the ECG electrodes were filtered and were amplified and were sent to an analog-to-digital converter (Power Lab data acquisition and analysis system: AD Instruments, Australia) attached to a computer. The signals recorded were saved for coming analysis. ECG were recorded and were analyzed with the help of LabChartpro7 software (AD Instruments, Australia).

The most important ECG parameters recorded were QRS, P, T voltage 'P-R, Q-T, RR, QTc, JT, TpTe intervals and $\mathrm{S}-\mathrm{T}$ segment elevation. These parameters were assessed by a cardiologist blinded of experiment. Using power lab (AD Instrument), electrocardiogram parameters were recorded for $2 \mathrm{~h}$ and then using lab chart software and ECG analysis module, the parameters were analysis. PR interval presents the atrial depolarization ( $\mathrm{P}$ wave) plus the duration of the electric current conduction through AV node and the specialized HisPurkinje system to the ventricles. In fact, P-R interval is the interval time from the onset of P-wave to onset of QRS complex (Q- or R wave) (Joukar and Asadipour, 2015).

The QT interval consists of both depolarization and repolarization of the heart and it is the interval time from the earliest $\mathrm{Q}$ or R-wave onset to the end of $\mathrm{T}$ wave. Using the Bazett's formula modified as QTen-B= QT/ $(\mathrm{RR} / \mathrm{f})^{1 / 2}$ which is a modified and applicable formula for QT correction in rat, corrected QT (QTc) interval dependence to heart rate was corrected. RR is R-R interval and $\mathrm{f}=150 \mathrm{~ms}$ (Joukar and Asadipour, 2015). In addition, $\mathrm{JT}$ interval as an interval from junction point $\mathrm{J}$ to $\mathrm{T}$ wave end and $\mathrm{T}$ peak to $\mathrm{T}$ end interval (TpTe), as the period from the peak of the $\mathrm{T}$ wave until the end of the $\mathrm{T}$ wave were computed. Beside this, the premature ventricular contraction (PVC), ventricular tachycardia (VT) and VF were computed during the $120 \mathrm{~min}$ of the experiment.

According to the Lambeth conventions, types of ventricular arrhythmias are defined as follows: ventricular premature beat (VPB; equal to $\mathrm{PVC}$ ) is defined as a ventricular complex (complete electrical event: QRS, RS, QRST or RST) that is different in shape (voltage, duration, height or width) from the preceding (non-VPB) ventricular complex, and is premature than 
the preceding ventricular complex and VT is a run of four or more ventricular premature beats. VF is defined as a sequence of a minimum of four consecutive ventricular complexes without intervening diastolic pauses in which the shape, the peak-peak interval, the height vary and the variation between each is non-progressive (Baravati et al., 2015).

In addition, the severity of arrhythmias in the different groups was evaluated quantitatively by Scoring system presented in the past. In each group, it was performed during $1 \mathrm{~h}$ of reperfusion and compared with control. This scoring was defined as follow: $0,<10$ PVCs; $1, \geq$ 10 PVCs; 2 , one to five episodes of VT; $3,>5$ episodes of VT or 1 episode of VF; 4, two to five episodes of VF; $5,>5$ episodes of VF; 6 , VT or VF or both with duration $>300 \mathrm{sec}$ (Baravati et al., 2015).

\section{Statistical analysis}

Using SPSS version 22, the results were analyzed using one-way ANOVA or repeated-measurement ANOVA followed by LSD multiple comparison test and expressed as mean \pm SEM. $P$-values of less than 0.05 were considered significant. Normality was checked using Kolmogorov Smirnov test. The ECG parameters changes between control group and others during the 2 hours of test were assessed. This time included the preischemia, ischemia and reperfusion period. During this time, we recorded the changes repeatedly. The main aim was to compare these changes between the control and treated groups. Comparison within each group was not made. For the severity of arrhythmias in the different groups, used Ki square test was used and the percentage was presented. All procedures were confirmed with ethical committee of Jahrom School of Medicine (ethical number: (JUMS.REC.1393.132)

\section{Results}

Data of this study showed that RR, JT, interval, p duration and $\mathrm{ST}$ elevation during ischemia period increased compared with pre-ischemia $(P<0.05)$; however, $\mathrm{P}, \mathrm{R}$, $\mathrm{S}, \mathrm{T}$ amplitudes were decreased in rats with ischemia in comparison with pre-ischemia or sham group $(P<0.05$, Tables 1and 2).

The QT, JT and TpTe intervals decreased significantly in animals which treated with combination of both drug $(\mathrm{G} 7.5+\mathrm{CsA} ; P<0.05)$. The PR interval reduced in rats which treated with combination of both drug
(G15+CsA) but $\mathrm{P}$ and $\mathrm{R}$ amplitudes increased in those animals (G15+CsA) compared with control group $(P<0.05)$. The $\mathrm{QRS}$ duration and $\mathrm{R}$ amplitude were increased in the animals which received combination of both drug $(\mathrm{G} 30+\mathrm{CsA})$ in comparison with control group, but QTC and TpTe intervals decreased in those group $(\mathrm{G} 30+\mathrm{CsA})$ rather than control $(P<0.05$, Table 1$)$. We checked the number of arrhythmias during 1 hour of reperfusion. In this case there were not a significant difference between groups which pretreatment with gallic acid and cyclosporine A in PAC, VT, QRS drop, $\mathrm{AVB}, \mathrm{PVC}$ and $\mathrm{CHB}$ in comparison with control group $(P=0.36$, Table 3$)$.

\section{Discussion}

The present was conducted to investigate and present the effects of combination of gallic acid and CsA on ECG parameters and arrhythmia prevalence after I/R injury in rat isolated hearts. The results showed that ECG parameters in control animals, including $\mathrm{p}$ duration, $\mathrm{RR}$ and JT interval were increased after reperfusion compared with sham group. In addition, $\mathrm{P}, \mathrm{R}, \mathrm{S}$ and $\mathrm{T}$ wave amplitudes were decreased during ischemia in control group compared with sham. The combination of gallic acid (15 and $30 \mathrm{mg} / \mathrm{kg}$ ) and cyclosporine shortened the PR and JT intervals and increased R, T and $P$ amplitudes.

ROS production increased and induced mitochondrial dysfunction and $\mathrm{Ca}$ overload because of increasing the permeability of transition pore in the mitochondrial membrane (Marin-Garcia and Goldenthal, 2004). Additional calcium can create the electrical changes and reduces the ATP synthesis in cardio myocytes (Rubart and Zipes, 2005). In addition, ischemia induces intracellular ATP reduction and inhibits the Na-K ATPase pump and gives rise to $\mathrm{Na}$ accumulation. After that, $\mathrm{Ca}$ overload occurred because of $\mathrm{Na}-\mathrm{Ca}$ exchanger reverse function and finally the expression of $\mathrm{K}$ channels decreased and action potential duration prolonged because of the major K efflux during hypoxia/ ischemia events via ATP dependent $\mathrm{K}$ channels and inward rectifier $\mathrm{K}$ channels (Rubart and Zipes, 2005). Furthermore, I/R injury caused the loss of voltage potassium channels function ( $\mathrm{Ik}_{\mathrm{s}}$, and $\mathrm{Ik}_{\mathrm{r}}$ ). Some researchers understood that gallic acid is a potent antioxidant and so it can restore the redox state. In the other side, CsA is an important mPTP inhibitor and preserves the mitochondria against oxidative stress 


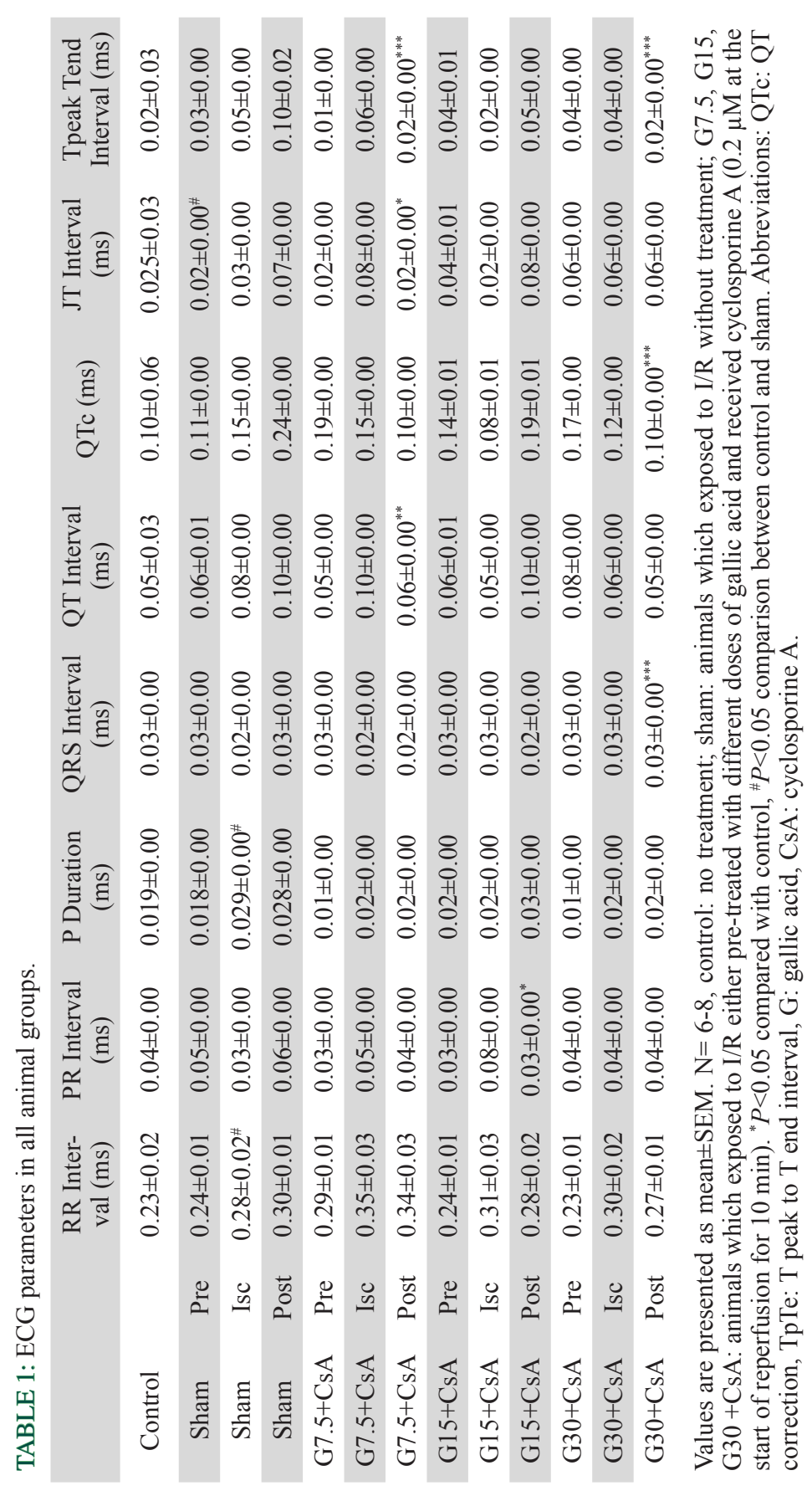

arising from ischemia and hypoxia.

This protection can maintain the ATP because the mitochondria is an origin organelle of ATP synthesis (Marin-Garcia and Goldenthal, 2004; Priscilla and Prince, 2009). We maintained the cardio myocytes against two disturbances factor (ROS and opening mPTP) capable of disordering the electrical balance in ischemic myocytes. The QRS complex indicates that $\mathrm{t}$ depolarization of ventricles and its amplitude may be reduced due to conduction system injuries because of ischemia (Joukar and Asadipour, 2015). The QTc interval shows the sum of depolarization and repolarization periods of ventricles. The JT interval is equal to point $\mathrm{J}$ to the $\mathrm{T}$ wave end and shows the ventricular repolarization. The TpTe is the mark of transmural repolarization of left ventricle (Zumbakyte-Sermuksniene et al., 2012). Pretreatment of animals with a combination of gallic acid (G7.5 and G15) and CsA (G7.5+CsA) shortened the JT interval.

We also investigated the hemodynamic changes of different doses of gallic acid in combination with CsA 
TABLE 2: Heart rate and ECG parameters in all animal groups

\begin{tabular}{ccccccccc}
\hline & & & $\begin{array}{c}\text { P Amplitude } \\
(\mathrm{mV})\end{array}$ & $\begin{array}{c}\text { Q Amplitude } \\
(\mathrm{mV})\end{array}$ & $\begin{array}{c}\text { R Amplitude } \\
(\mathrm{mV})\end{array}$ & $\begin{array}{c}\text { S Amplitude } \\
(\mathrm{mV})\end{array}$ & $\begin{array}{c}\text { ST Height } \\
(\mathrm{mV})\end{array}$ & $\begin{array}{c}\text { T Amplitude } \\
(\mathrm{mV})\end{array}$ \\
Control & & $273.23 \pm 22.90$ & $1 \pm 0.16$ & $-0.027 \pm 0.69$ & $1.71 \pm 0.00$ & $-0.5 \pm 0.092$ & 0 & $1.42 \pm 0.47$ \\
Sham & Pre & $244.64 \pm 10.54$ & $0.71 \pm 0.16$ & $-0.02 \pm 0.00$ & $1.57 \pm 0.091$ & $-0.5 \pm 0.035$ & 0 & $1.07 \pm 0.01$ \\
\hline Sham & Isc & $264.87 \pm 5.63$ & $-0.03 \pm 0.00^{\#}$ & $-0.08 \pm 0.00$ & $0.78 \pm 0.00^{\#}$ & $-0.10 \pm 0.00^{\#}$ & $0.36 \pm 0.00^{\#}$ & $-0.06 \pm 0.00^{\#}$ \\
Sham & Post & $207.93 \pm 13.64$ & $-0.18 \pm 0.00$ & $-0.07 \pm 0.03$ & $3.26 \pm 0.57$ & $-1.52 \pm 0.12$ & $1.44 \pm 0.51$ & $0.21 \pm 0.046$ \\
\hline G7.5+CsA & Pre & $212.58 \pm 9.19$ & $0.8 \pm 0.00$ & $-0.054 \pm 0.017$ & $1.2 \pm 0.060$ & $-0.32 \pm 0.00$ & 0 & $1.00 \pm 0.00$ \\
G7.5+CsA & Isc & $190.65 \pm 16.34$ & $-0.09 \pm 0.00$ & $-0.13 \pm 0.03$ & $0.51 \pm 0.12$ & $-0.20 \pm 0.10$ & $0.35 \pm 0.11$ & $-0.41 \pm 0.12$ \\
\hline G7.5+CsA & Post & $186.96 \pm 14.13$ & $0.01 \pm 0.00$ & $-0.53 \pm 0.15$ & $1.4 \pm 0.63$ & $-0.26 \pm 0.05$ & $1.18 \pm 0.43$ & $0.93 \pm 0.21$ \\
\hline G15+CsA & Pre & $249.58 \pm 8.62$ & $0.6 \pm 0.06$ & $-0.085 \pm 0.024$ & $1.4 \pm 0.040$ & $-0.51 \pm 0.14$ & 0 & $1.12 \pm 0.12$ \\
\hline G15+CsA & Isc & $209.28 \pm 15.32$ & $-0.03 \pm 0.00$ & $-0.21 \pm 0.08$ & $0.37 \pm 0.09$ & $-0.26 \pm 0.08$ & $0.22 \pm 0.020$ & $-0.36 \pm 0.028$ \\
\hline G15+CsA & Post & $225.21 \pm 15.26$ & $0.09 \pm 0.01^{* * *}$ & $-0.025 \pm 0.17$ & $1.15 \pm 0.22^{* *}$ & $-0.40 \pm 0.19$ & $0.83 \pm 0.20$ & $0.18 \pm 0.015$ \\
\hline G30+CsA & Pre & $259.47 \pm 7.97$ & $1 \pm 0.00$ & $-0.013 \pm 0.003$ & $2 \pm 0.58$ & $-0.60 \pm 0.00$ & 0 & $1.18 \pm 0.28$ \\
\hline G30+CsA & Isc & $206.49 \pm 12.15$ & $-0.01 \pm 0.00$ & $-0.22 \pm 0.00$ & $0.62 \pm 0.15$ & $-0.21 \pm 0.00$ & $0.17 \pm 0.017$ & $-0.47 \pm 0.022$ \\
\hline G30+CsA & Post & $222.58 \pm 9.79$ & $0.01 \pm 0.00$ & $-0.21 \pm 0.04$ & $1.14 \pm 0.15^{* *}$ & $-0.77 \pm 0.00$ & $1.06 \pm 0.52$ & $-0.31 \pm 0.035$ \\
\hline
\end{tabular}

Values are presented as mean $\pm \mathrm{SEM}$. $\mathrm{N}=6-8$, control: no treatment, sham: animals which exposed to I/R without treatment; G7.5, G15, G30 + CsA: animals which exposed to I/R either pretreat with different doses of gallic acid and received by cyclosporine A $(0.2 \mu \mathrm{M}$ at the start of reperfusion for $10 \mathrm{~min}) .{ }^{*} P<0.05$ compared with control, ${ }^{\#} P<0.05$ comparison between control and sham. Abbreviations: HR: heart rate, BPM: beat per minute, G: gallic acid, CsA: cyclosporine A.

TABLE 3: Percentage of arrhythmia in experimental groups.

\begin{tabular}{|c|c|c|c|c|c|c|}
\hline & $\mathrm{PAC} \%$ & VT\% & PVC\% & AVB $\%$ & QRS drop $\%$ & $\mathrm{CHB} \%$ \\
\hline CsA & 13.8 & 15.2 & 12.7 & 16.7 & 3.4 & 12.5 \\
\hline G7.5 & 12.1 & 13 & 12.7 & 16.7 & 24.1 & 15.6 \\
\hline G7.5+CsA & 13.8 & 13 & 15.7 & 12.5 & 10.3 & 12.5 \\
\hline G15 & 12.1 & 15.2 & 12.7 & 20.8 & 17.2 & 9.4 \\
\hline G15+CsA & 13.8 & 15.2 & 20.7 & 8.3 & 13.8 & 15.6 \\
\hline G30 & 10.3 & 13 & 9.1 & 8.3 & 13.8 & 9.4 \\
\hline G30+CsA & 12.1 & 8.7 & 14.5 & 0 & 10.3 & 18.8 \\
\hline Control & 12.1 & 6.5 & 6.7 & 16.7 & 6.9 & 6.3 \\
\hline P value & 0.5 & 0.5 & 0.049 & 0.2 & 0.08 & 0.057 \\
\hline
\end{tabular}

G: gallic acid, CsA: cyclosporine A

and found that the best dose was G15+CsA (Badavi et al., 2017). In this section, the low and medium dose (G7.5 and G15) were better than high dose. Therefore, it was possible that (G7.5 and G15) doses had antioxidant properties but at high dose, the antioxidant entity became less. To amplify this idea, Shinno et al. (2015) proved the high dose of gallic acid have prooxidant specifications (Shinno et al., 2005).

One study reported that PR interval was prolonged and AV block was increased during anoxia and reoxygenation due to alteration in cell to cell communication (Sarre et al., 2005). In agreement with our study, Sun et al. (2013) demonstrated that QT prolongation occurred during the myocardial infarction. In addition, pretreatment with combination of gallic acid at dose of $15.30 \mathrm{mg} /$ $\mathrm{kg}$ and CsA (G15.30+CsA) decreased the PR, QTc and TpTe intervals in rats' hearts undergone the ischemia compared with control group. In accordance with previous study (Joukar et al., 2010). In the groups exposed to ischemia, $\mathrm{P}, \mathrm{R}, \mathrm{S}$ and $\mathrm{T}$ wave amplitudes decreased and pretreatment with combination of gallic acid at dose of $15.30 \mathrm{mg} / \mathrm{kg}$ and CsA (G15.30+CsA), increased $\mathrm{P}$ and $\mathrm{R}$ waves amplitude during reperfusion time. These alterations can be attributed to reduction of AV node or His purkinje system conduction induced by degeneration of conduction pathways due to ischemia (Rubart and Zipes, 2005).

Along with this result, other researchers founded that loss of $\mathrm{K}^{+}$and its extracellular accumulation during myocardial ischemia is responsible to sustained 
membrane depolarization and leads to conduction slowing and VT/VF promotion (Rubart and Zipes, 2005). We also checked the number of arrhythmias during 1 hour of reperfusion. In this case, there was not a significant difference between the groups in PAC, VT, QRS drop, AVB and CHB, but pretreatment with gallic acid at dose $30 \mathrm{mg} / \mathrm{kg}$ caused significant decrease in the PVC number. The reasons of arrhythmias induction after $\mathrm{I} / \mathrm{R}$ is remodeling of cardiac tissue including structural myocardial modifications or abnormality of myofibrils or collagens (Dianat et al., 2014). During ischemia, the alteration of electrical homogeneity of heart can provides a condition of reentry phenomenon and facilitates ventricular arrhythmias (Anderson et al., 2006) or myocardial remodeling (Yang et al., 2011) which can increase the VF susceptibility.

In addition, an increase of intracellular calcium can elevate the susceptibility to arrhythmia (Estrada et al., 2003). In accordance with previous reports, high calcium concentration induced by high number of depolarizations lead to extra heart beats and as a result, tendency to cardio myocytes (DAD) increases and at this time, arrythmias are induced (Estrada et al., 2003). The known probably mechanism is that the calcium wave through activation of $\mathrm{Ca}^{+2}$ sensitive inward currents will depolarize the DAD. This current activated the inward $\mathrm{I}_{\mathrm{Na}}^{+}+{ }^{+}{ }^{+2}$ as the major candidate for transient current underlying DADs. If magnitude of DAD is sufficient, it will depolarize cardio myocyte above threshold resulting into a single or repetitive premature beats capable of commencing the arrhythmia (Rubart and Zipes, 2005). Priscilla et al. showed that that gallic acid can protect the lysosomal membrane against isoproterenol cardiac damage (Priscilla and Prince, 2009). Along with this result, in the present study, the gallic acid probably prevents the calcium overload through protecting mitochondria membrane. It should be noted that because of the limitations of working with animals including ECG recordings this study was done in lead II alone. Therefore, clarification of involved mechanisms demands complementary studies.

\section{Conclusion}

Pretreatment with a combination of both drugs shortened the QT and JT intervals and increased the R and $\mathrm{T}$ amplitude. Gallic acid at doses of 15 and 30mg/ $\mathrm{kg}$ acted better than other doses and protected the cardio myocytes against ischemia induced electrical changes. In addition, RR and PR intervals didn't change. Therefore, pretreatment with a combination gallic acid and CsA was more effective on ventricular electrical activity rather than atrial.

\section{Acknowledgements}

The authors express their thanks to Ahvaz Physiology Research Center as well as Jahrom Medical University for their collaboration. The data presented in this article were derived from a student of medicine thesis (Arman khorram) performed in the Department of Physiology and Research Center of School of Medicine, Jahrom University of Medical Sciences, and Ahvaz Physiology Research Center, Iran.

\section{Conflict of interests}

None declared

\section{References}

Adib A, Masoompour SM, Vardanjani HM, Gondomkar A, Poustchi H, Salehi A, et al. Smoking water-pipe, opium Use and prevalence of heart disease: a cross-sectional analysis of baseline data from the Pars Cohort Study, Southern Iran. Arch Iran Med 2020; 23: 289-95. https://doi.org/10.34172/ aim.2020.17

Anderson CL, Delisle BP, Anson BD, Kilby JA, Will ML, Tester DJ, et al. Most LQT2 mutations reduce Kv11. 1 (hERG) current by a class 2 (trafficking-deficient) mechanism. Circulation 2006; 113: 365-73. https://doi.org/10.1161/CIRCULATIONAHA.105.570200

Badavi, M, Sadeghi, N, Dianat, M, Samarbafzadeh A. Effects of gallic Acid and cyclosporine a on antioxidant capacity and cardiac markers of rat isolated heart after ischemia/ reperfusion. Iran Red Crescent Med J 2014; 16: e16424. https://doi.org/ 10.5812/ircmj.16424

Badavi M, Sadeghi N, Dianat M, Samarbafzadeh A. Gallic acid and cyclosporine mixture and their effects on cardiac dysfunction induced by ischemia/reperfusion and eNOS/ iNOS expression. Int J Cardiovasc Sci 2017; 30: 207-18. https://doi.org/10.5935/2359-4802.20170047

Baravati HG, Joukar S, Fathpour H, Kordestani Z. Nandrolone plus moderate exercise increases the susceptibility to lethal arrhythmias. Res Cardiovasc Med 2015; 4. https:// doi.org/10.4103/2251-9572.218777

Buja LM, Weerasinghe P. Unresolved issues in myocardial reperfusion injury. Cardiovasc Pathol 2010; 19: 29-35. 
https://doi.org/10.1016/j.carpath.2008.10.001

Dianat M, Akbari GH, Badavi M. Antidysrhythmic effects of gallic acid on cacl2-induced arrhythmia in rat. Int J Res Dev Pharm L Sc 2013; 2: 686-9.

Dianat M, Sadeghi N, Badavi M, Panahi M, Moghadam MT. Protective effects of co-administration of gallic acid and cyclosporine on rat myocardial morphology against ischemia/ reperfusion. Jundishapur J Nat Pharm Prod 2014; 9. https:// doi.org/10.17795/jjnpp-17186

Ebner F. Carbon dioxide, oxygen, and serum biomarkers after out-of-hospital cardiac arrest. Lund University, Faculty of Medicine Doctoral Dissertation Series 2020.

Estrada M, Espinosa A, Müller M, Jaimovich E. Testosterone stimulates intracellular calcium release and mitogen-activated protein kinases via a $G$ protein-coupled receptor in skeletal muscle cells. Endocrinology 2003; 144: 3586-97. https://doi.org/10.1210/en.2002-0164

Giugliano D. Dietary antioxidants for cardiovascular prevention. Nutr Metab Cardiovasc Dis 2000; 10: 38-44.

Hausenloy DJ, Duchen MR, Yellon DM. Inhibiting mitochondrial permeability transition pore opening at reperfusion protects against ischaemia-reperfusion injury. Cardiovasc Res 2003; 60: 617-25. https://doi.org/10.1016/j.cardiores.2003.09.025

Hung LM, Chen JK, Lee RS, Liang HC, Su MJ. Beneficial effects of astringinin, a resveratrol analogue, on the ischemia and reperfusion damage in rat heart. Free Radic Biol Med 2001; 30: 877-83. https://doi.org/10.1016/S08915849(01)00474-9

Joukar S, Asadipour H. Evaluation of Melissa officinalis (lemon balm) effects on heart electrical system. Res Cardiovasc Med 2015; 4. https://doi.org/10.5812/cardiovascmed.4(2)2015.27013

Joukar S, Najafipour H, Malekpour-Afshar R, Mirzaeipour F, Nasri HR. The effect of passive opium smoking on cardiovascular indices of rabbits with normal and ischemic hearts. Open Cardiovasc Med J 2010; 4: 1. https://doi.org/10.2174/1874192401004010001

Kario K. Measuring the effects of stress on the cardiovascular system during a disaster: the effective use of self-measured blood pressure monitoring. J Hypertens 2010; 28: 657-9. https://doi.org/10.1097/HJH.0b013e32833815a4

Klawitter PF, Murray HN, Clanton TL, Angelos MG. Reactive oxygen species generated during myocardial ischemia enable energetic recovery during reperfusion. Am J Physiol Heart Circ Physiol 2002; 283: H1656-61. https://doi.org/10.1152/ajpheart.00041.2002
Marin-Garcia J, Goldenthal MJ. Heart mitochondria signaling pathways: appraisal of an emerging field. J Mol Med 2004; 82: 565-78. https://doi.org/10.1007/s00109-0040567-7

Moradi-Lakeh M, Kiadaliri AA, Asadi-Lari M, Asayesh H, Esteghamati AR, Farvid MS, et al. Trend of socio-demographic index and mortality estimates in iran and its neighbors, 1990-2015; Findings of the global burden of diseases 2015 study. Arch Iran Med 2017; 20.

Pagliaro P, Femminò S, Penna C. Redox aspects of myocardial ischemia/reperfusion injury and cardioprotection. In Chakraborti S, Dhalla NS, Ganguly NK, Dikshit M. Oxidative Stress in Heart Diseases. Springer Singapore: Singapore, 2019, p. 289-324. https://doi.org/10.1007/978-98113-8273-4_13

Prince PS, Priscilla H, Devika PT. Gallic acid prevents lysosomal damage in isoproterenol induced cardiotoxicity in Wistar rats. Eur J Pharmacol 2009; 615: 139-43. https://doi. org/10.1016/j.ejphar.2009.05.003

Priscilla DH, Prince PS. Cardioprotective effect of gllic acid on cardiac troponin-T, cardiac marker enzymes, lipid peroxidation products and antioxidants in experimentally induced myocardial infarction in Wistar rats. Chem Biol Interact 2009; 179: 118-24. https://doi.org/10.1016/j. cbi.2008.12.012

Rubart M, Zipes DP. Mechanisms of sudden cardiac death. J Clin Invest 2005; 115: 2305-15. https://doi.org/10.1172/ JCI26381

Sarre A, Lange N, Kucera P, Raddatz E. mitoKATP channel activation in the postanoxic developing heart protects E-C coupling via NO-, ROS-, and PKC-dependent pathways. Am J Physiol Heart Circ Physiol 2005; 288: H1611-9. https://doi.org/10.1152/ajpheart.00942.2004

Shinno E, Shimoji M, Imaizumi N, Kinoshita S, Sunakawa $\mathrm{H}$, Aniya Y. Activation of rat liver microsomal glutathione S-transferase by gallic acid. Life Sci 2005; 78: 99-106. https://doi.org/10.1016/j.lfs.2005.04.034

Sun X, Cai J, Fan X, Han P, Xie Y, Chen J, et al. Decreases in electrocardiographic R-wave amplitude and QT interval predict myocardial ischemic infarction in Rhesus monkeys with left anterior descending artery ligation. PLoS One 2013; 8: e71876. https://doi.org/10.1371/journal. pone. 0071876

White C. Protein phosphorylation in Ischaemia and reperfusion of the heart: a focus on protein phosphatase. Thesis (PhD)--Stellenbosch University, 2020.

Xie JR, Yu LN. Cardioprotective effects of cyclosporine A in 
an in vivo model of myocardial ischemia and reperfusion. Acta Anaesthesiologica Scandinavica 2007; 51: 909-13. https://doi.org/10.1111/j.1399-6576.2007.01342.x

Yang P, Han P, Hou J, Zhang L, Song H, Xie Y, et al. Electrocardiographic characterization of rhesus monkey model of ischemic myocardial infarction induced by left anterior descending artery ligation. Cardiovasc Toxicol 2011; 11: 365. https://doi.org/10.1007/s12012-011-9129-8

Yellon DM, Hausenloy DJ. Myocardial reperfusion injury. N Engl J Med 2007; 357: 1121-35. https://doi.org/10.1056/ NEJMra071667
Zheng J, Chu H, Struppa D, Zhang J, Yacoub SM, El-Askary $\mathrm{H}$, et al. Optimal multi-Ssage arrhythmia classification approach. Sci Rep 2020; 10: 1-7. https://doi.org/10.1038/ s41598-020-59821-7

Žumbakytė-Šermukšnienė R, Kajènienè A, Berškienè K, DaunoravičienėA, Sederevičiūtè R. Assessment of the effect of anthropometric data on the alterations of cardiovascular parameters in Lithuanian elite male basketball players during physical load. Medicina 2012; 48: 83. https://doi. org/10.3390/medicina48110083 